\title{
Hybrid Evolutionary Algorithm Using Optimal Placement of FACTS Devices for Total Transfer Capability
}

\author{
Gujjula Ganesh', Ch.Narendrakumar² \& Ezhil Vignesh.K ${ }^{3}$ \\ ${ }^{1-3}$ Department of Electrical \& Electronics Engineering, Malla Reddy Engineering College (A), Hyderabad, Telangana, India. \\ Email: ganeshgujjula920@gmail.com ${ }^{1}$, chnarendrakumar@gmail.com² \&ezhilvigneshk@gmail.com ${ }^{3}$
}

DOI: $10.46382 / M J B A S .2020 .4208$

The paper discusses about a hybrid model with an evolutionary algorithm (HEA) for identifying the multi-type flexible AC transmission systems (FACTS) procedures to improve the total transfer capability (TTC). To reduce the loss of power this transferences among various control regions. FACTS devices with Multi objective optimal power flow (OPF) which include TTC to determine a reasonable value without violating system limitations. The results are simulated for FACTS devices with the HEA algorithm which emerges TTC value using an efficient methods using conventional transmission system. The simulation results are obtained by MATLAB/SIMULINK environment.

\section{Introduction}

In recent power systems, the applications concerned with power transmission techniques are developed repetitively. Flexible AC transmission systems (FACTS), have been used for controlling the flow of power, enhance stability of transmission, and increase the safety in power transmission system [1]. Additionally, these devices could be maximized power transfer capability \& minimized power loss of the transmission systems, which lead for the effective applications compared to the conventional power system. In physical transmission, Available transfer capability (ATC) is used for transferring the capacity in a transmission network. This needs to be measured for every control region which is given in societal problems for enhancing the open acceptance in a power system.

ATC is known as the total transfer capability (TTC) which reduces the transmission reliability margin (TRM), capacity benefit margin (CBM) and the conventional transmission commitments (CTC). The TTC is an important part at ATC calculation. TTC is called the number electrical power which could reassign connected transmission network in a dependable. Huge categories of techniques, like continuation power flow (CPF) [4], linear ATC (LATC) [3], and repetitive power flow (RPF) [5] strategies are introduced to manipulate TTC. Moreover, optimal power flow (OPF) techniques that are developed using several optimizing strategies $[6,7]$, are introduced for TTC controls several degrees. The technique which needs to find the operations to identify correct solution. Therefore, OPF is a nonlinear function \& non-convex optimizing method which results in many solutions that exist the special function in power networks with FACTS devices [8]. The device factors have extra control enables which could not be solved using existing OPF methods due to the factors will 
alter the impedance. However, existing optimizing techniques are used for local optimal solutions. Recently, the power transfer capability improvement [10] \& power loss minimization technique by multi-type FACTS devices. Due to these improve competition, minimize operating costs, and effectively apply the conventional power transmission systems. In addition to the advantages the compatibility of the system how they are controlled with other systems. Therefore, the techniques lead to the correct solution due to the loading conditions. GA is searching the exact location of TCSC and CPF is involved to examine the FACTS devices relevant to thermal limits and voltage limits.

\section{Problem Statement}

The devices includes TTC, loss of power in the transmission system and along with Multi-objective OPF FACTS devices are designed to find TTC value which will be moved through a range of generator set for connecting loads. And it has various limits such as reactive power voltage limits, generation limits, thermal limits, FACTS operation limits and steady state stability limits.

FACTS devices are categorized into 4 parts as follows:

\section{TCSC}

2. Unified power flow controller (UPFC)

3. Thyristor-controlled phase shifter (TCPS)

4. SVC.

Maximize

$$
T=\sum_{i=1}^{N D_{-} S N K} P_{D i}-\sum_{i=1}^{N}\left(P_{G i}-P_{D i}\right)-P F
$$

Subject to

$$
\begin{aligned}
& P_{G i}-P_{D i}+\sum_{k=1}^{m(i)} P_{P i}\left(\alpha_{P k}\right)+\sum_{k=1}^{n(i)} P_{U i}\left(V_{U k}, \alpha_{U k}\right) \\
& -\sum_{j=1}^{N} V_{i} V_{j} Y_{i j}\left(X_{S}\right) \cos \left(\theta_{i j}\left(X_{S}\right)-\delta_{i}+\delta_{j}\right)=0
\end{aligned}
$$

Where

$\mathrm{T}$ objective function

$\mathrm{PF}$ penalty function 
$\mathrm{P}_{\mathrm{Gi}}^{\min }, \mathrm{P}_{\mathrm{Gi}}^{\max }$ real power generation in lower and upper limits at bus $\mathrm{i}$

$\mathrm{Q}^{\min } \mathrm{Gi}, \mathrm{P}^{\max } \mathrm{Gi}$ reactive power generation in lower and upper limits at bus $\mathrm{i}$

$V^{\min }, V^{\max }{ }_{i}$ voltage magnitude in lower and upper limits of bus $i$

$S^{\max }{ }_{L i} i$ th line or transformer loading limit

$\mathrm{I}^{\text {crit }}{ }_{\mathrm{ij}}$ critical angle difference between bus $\mathrm{i}-\mathrm{j}$

$\mathrm{X}^{\min } \mathrm{Si}, \mathrm{X}^{\max } \mathrm{si}$ lower and upper limits of TCSC at line $\mathrm{i}$

$a^{\max }{ }_{\mathrm{Pi}}, \mathrm{a}_{\mathrm{Pi}}^{\max }$ TCPS lower and upper limits in line $\mathrm{i}$

$V_{\text {min }}^{\min } \mathrm{V}_{\text {Ui }}^{\max }$ UPFC of lower and upper limits at line $i$

$a^{\min }$ Ui,$a^{\max }$ Ui UPFC lower and upper limits at line $i$

$\mathrm{Q}^{\min } v_{\mathrm{i}}, \mathrm{Q}^{\max } v_{\mathrm{i}}$ reactive power injected in SVC at bus $\mathrm{i}$

$\mathrm{N}, \mathrm{NL}$ amount buses and branches

NG, ND amount load buses

NG_SCE amount of source area

ND_SNK amount of load buses in a sink area

$P_{G i}^{\min } \leq P_{G i} \leq P_{G i}^{\max } \quad \forall i \in N G$

$Q_{G i}^{\min } \leq Q_{G i} \leq Q_{G i}^{\max } \quad \forall i \in N G$

$V^{\min } \leq V_{i} \leq V^{\max }{ }_{i} \quad \forall i \in N$

$\left|S_{L i}\right| \leq S_{L i}^{\max } \forall i \in N \mathrm{~L}$

$V C P I_{i} \leq 1 \forall i \in N$

$\left|\delta_{I J}\right| \leq \delta^{c r i t}{ }_{i j} \forall i \in N L$

$X_{S i}^{\min } \leq X_{S i} \leq X_{S i}^{\max } \forall i \in N$

$\alpha_{P i}^{\min } \leq \alpha_{P i} \leq \alpha_{S i}^{\max }$

$V_{U i}^{\min } \leq V_{U i} \leq V_{U i}^{\max }$

$\alpha_{U i}^{\min } \leq \alpha_{U i} \leq \alpha_{U i}^{\max }$

$Q_{V i}^{\min } \leq Q_{V i} \leq Q_{V i}^{\max } \quad \forall i \in N G$

Variable series reactance model is subdivided into TCPS, UPFC, and SVC by power model of injected described in Appendix A [19]. 
In order to enhance EC methods, Hybrid Evolutionary Algorithm (HEA) is introduced along with TS, EP, and SA strategies. Advantages of the HEA algorithm are stated as follows:

Multiple populations along with modification operatives are developed for improving search and increase population update, providing greater caliber of provisions when compared to conventional searching methods.

The procedure is completed for transform and fuse the information contains sub-data assembly brought about by consistency of people in a solitary populace will be eased.

Choice of probabilistic changing method dependent on annealing schedule of SA and TS is given to remove need of operating functions \& to get local optimal explanations.

The procedure will certainly simplify similar execution of parallel computers for minimizing the lapsed time foregoing caliber of results.

Four categories in FACTS devices of reasonable every type, that allocates the input information. Position of the system is considered as 3 boundaries: nCF k, ' $k$ ' location, and ' $k$ ' parameter are considered as load (21).

FACTs devices category is I $€\{1,2,3,4\}$ involving placement of TCSC, TCPS, UPFC, and SVC, separately, the quantity of FACTs device category I, nCF I 2 fo; $1 \mathrm{~g}$. Obviously, none of

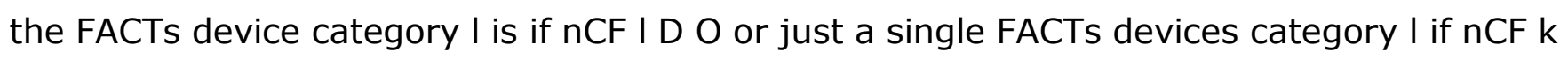
D I. Along these lines, plenty of FACTs parts, areas, \& boundaries of every class FACTs devices at the same time are used with the HEA calculation. All boundaries in FACTs device category $\mathrm{k}$ is legitimate just if $\mathrm{nCF}$ I D I.

This system is shown in Figure. It is divided as three regions, each has 2 generators. Updated system datasets are specified in [25]. A bilateral transaction has double transactions including from 1 bus to 21 bus $\&$ a multilateral transaction starting at region 1 and 2 with the following objectives:

Increase TTC, decrease power loss, increase TTC and low loss

Through 1 bus to 21 by not considering FACTs devices Table 1, in bilateral transaction the load values is $17.50 \mathrm{MW}$ in bus-21. To increase TTC by the proposed strategy, TTC value is taken as $40.447 \mathrm{MW}$ by not altering limits, are $0.84 \%, 1.29 \%, 0.58 \%$, and $0.31 \%$ through TS, EP, IEP and TS/SA respectively. For minimizing the power loss the current productions and load, bus voltages in generators are improved by adapting methods like TTC, HEA, and power losses is 2.045 and 17.50 Mega Watt, as expected. TTC increase and diminish loss by $\mathrm{HEA}$, TTC is $40.449 \mathrm{MW}$, that are greater than through EP-0.85\%, TS-0.55\%, TS/SA-0.38\%, 
and IEP- $0.58 \%$. The proposed devices are used to concurrently increase TTC and reduce the loss.

TTC has the value of 154.061 Mega Watt by not considering the limits that can be maximized at $280.88 \%$ when comparatively with FACTS devices. Moreover, TTC is from EP-22.25\%, TS-21.54\%, TS/SA-20.91\%, and IEP-15.04\% methods. To TTC increase or lossless, generally these are developed for improving the TTC and to eliminate power comparatively OPF when not considering the FACTs devices. In Parallel, improve the TTC and decrease the power by HEA technique, TTC is 125.930 Mega Watt, that are more from EP0.21\%, TS- $0.12 \%$, TS/SA-0.10\%, and IEP- $0.17 \%$ methods. In HEA algorithm also use a source region, growth of output power, and novel improvement in production bus voltages.

In parallel HEA placed every category of FACTs devices are improving TTC and minimizing loss. TTC has 191.379 Mega Watt, which increases $51.97 \%$ comparatively than not considering FACTS devices. Additionally, the TTC value is, more EP-40.68\%, TS-20.60\%, TS/SA-18.40\%, and IEP-15.61\% methods.

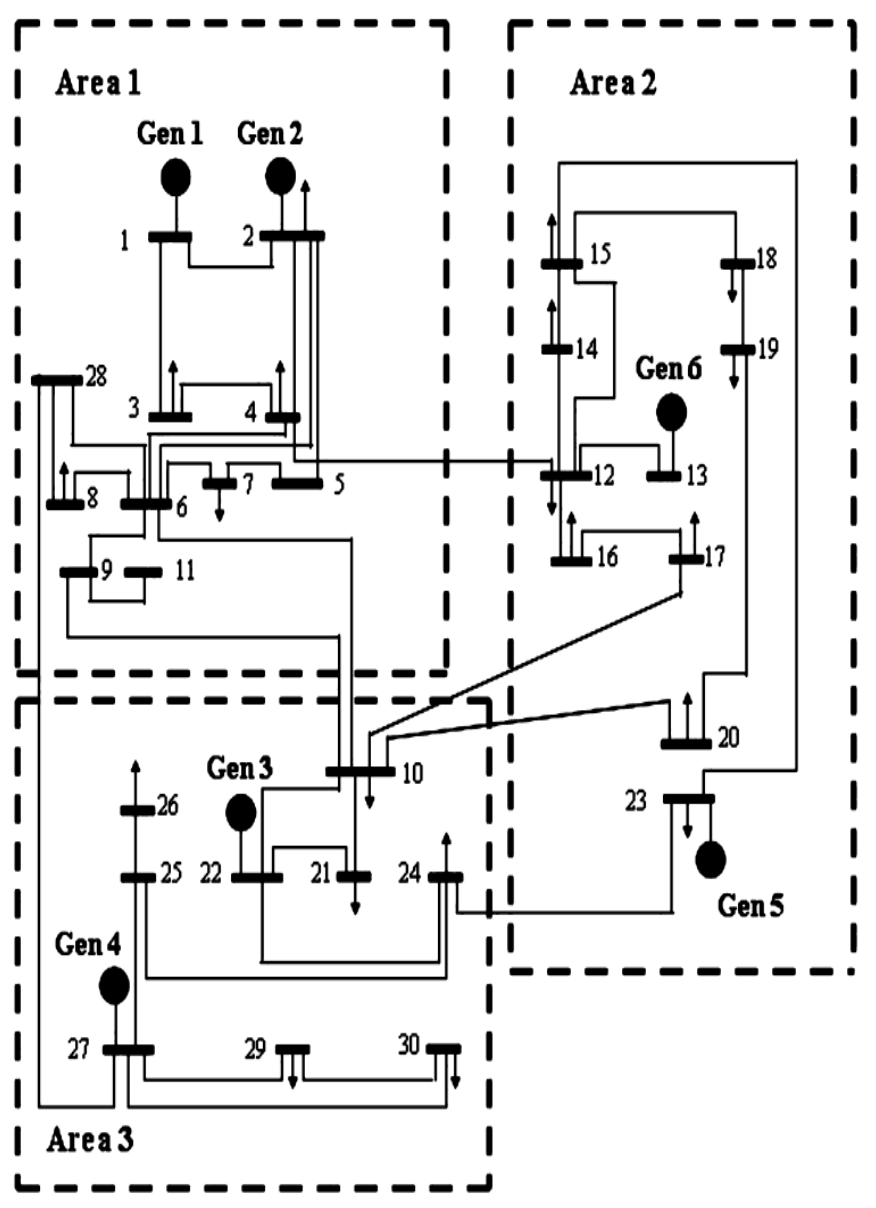

Figure.1 Modified IEEE-30 bus
CPU execution period is the over-all computation time for HEA algorithm beginning to final includes the NR power flow is shown in Figure 4. Using HEA method, results are obtained on the optimized values through various methods due to the selection mechanism of HEA algorithm with an updating approach depends on SA and TS algorithms to reduce the operational set-up corrected values. Hence, the variations of HEA is best solution as small as demonstrated in solutions, which leads for higher stability of HEA method.

\section{The Modified IEEE 118-Bus System}

It has 54 numbers of bus generators and 186 branches. It is divided as nine regions, as stated in Figure 5. Thermal limits are specified in [25] and [26], correspondingly. The dataset is improved as follows. Power 
production has the upper limit in 69 bus is 1,000 Mega Watt. Power production for reactive is upper limits starting from bus 34, 70, and ends in 103 are 80 MVAr. Power production minimum limit is bus $19,32,34,102$, and 105 is 22 MVAr. The Thermal limit at line 65-66 is $300 \mathrm{VA}$.

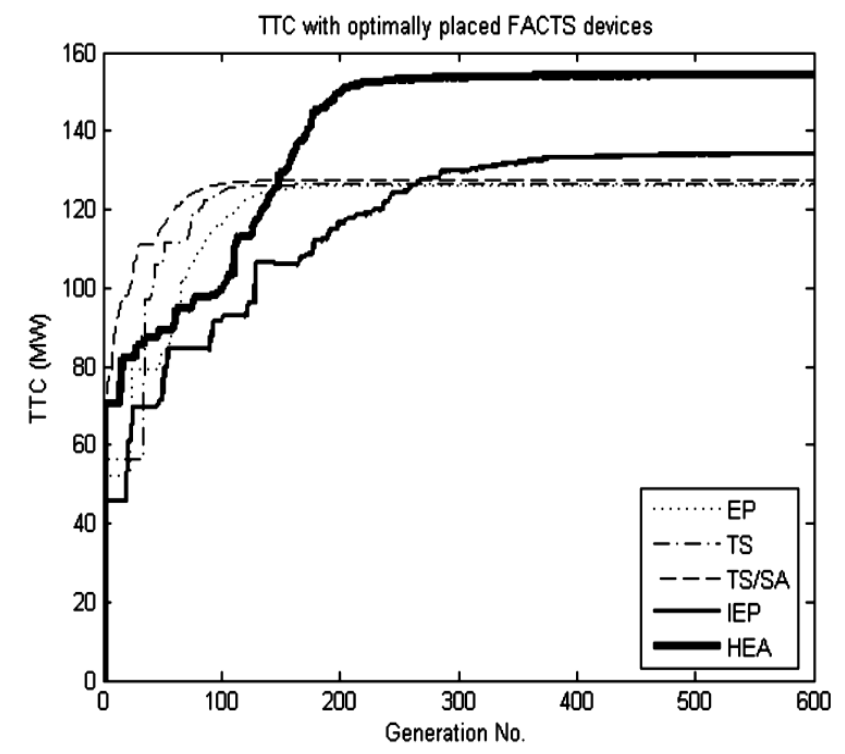

Figure. 2 Characteristics of solutions

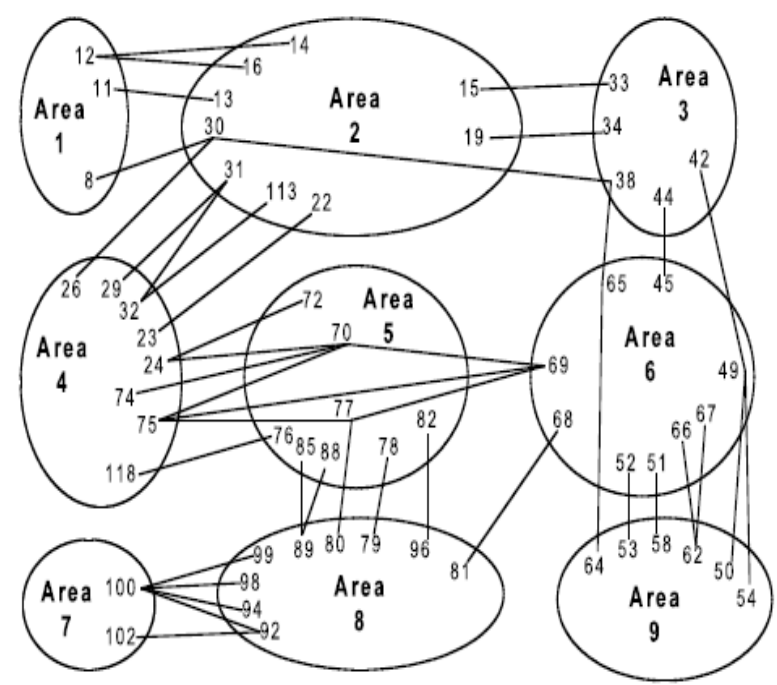

Figure.3 Control areas of the modified IEEE 118-bus system

The ML from region 6 to 3 with contingency constraints is considered. Output of the largest generators for each region and the output are included in the contingency list. Load in region 6 is 406.00 MegaWatt and the system real-power loss is 132.863 MegaWatt. TTC when not considering FACTS devices using HEA method is 710.57 MegaWatt. To find the pre-specified contingency controls are as shown in Table 5, TTC value using HEA algorithm is 461.03 MegaWatt without using network components, which is, from EP-4.89\%, TS-5.25\%, TS/SA-0.91\%, and IEP-0.57\%. Additionally, TTC value is minimized by $35.12 \%$ comparatively greater by not considering contingency components. Factor has the connected line from 42-49 among two regions are output. It is explained that rejecting the impact of given constraints on TTC is found insecure system operation. In parallel, to improve the TTC and lossless system in HEA has optimally placed every category of FACTS devices. TTC value FACTs devices is $725.17 \mathrm{MW}$, which is maximized by $2.05 \%$ compared to that without FACTs devices.

TTC value using HEA is 513.6 MegaWatt that increases $11.41 \%$ comparatively not considering FACTS devices. The interconnected line is 38-65 output among these regions. However, the TTC value is more than from EP-6.77\%, TS-7.93\%, TS/SA-5.26\%, and 
IEP-4.08\%. Table 4 states the corrected placement of multi-type FACTS devices for the TTC values. Simulations are indicated in Table 4 that of EP, TS/SA, and TS, is low efficient than population search of HEA and IEP techniques. Since, the HEA calculation needs greater operation period, for scheduling horizon, the calibre of solutions is high significant.

Table 1: Simulation Results (The Modified IEEE 30-Bus System)

\begin{tabular}{|l|c|c|c|c|c|c|}
\hline \multicolumn{7}{|c|}{ Without FACTS devices } \\
\hline \multicolumn{2}{|c|}{ Maximize TTC } & $\begin{array}{c}\text { Minimize } \\
\text { loss }\end{array}$ & min. loss \\
\hline Method & TTC & LosS & TTC & Loss & TTC & Loss \\
\hline EP & 124.994 & 6.421 & 56.200 & 2.029 & 125.663 & 6.035 \\
\hline TS & 125.553 & 6.140 & 56.200 & 2.029 & 125.781 & 5.916 \\
\hline TS/SA & 125.808 & 6.287 & 56.200 & 2.029 & 125.806 & 5.793 \\
\hline HEP & 125.451 & 6.248 & 56.200 & 2.029 & 125.716 & 5.967 \\
\hline
\end{tabular}

\section{Conclusion}

In this paper, the HEA algorithm is designed to find the optimal placement of FACTs device in multi-type by paralleled increasing TTC value and decreasing the power loss in power transactions among different control regions. The results are obtained for the placement OPF FACTs devices through HEA algorithm. This will increase the TTC value based on the normal and contingency conditions in the proposed system.

\begin{tabular}{|l|c|c|c|c|c|c|}
\hline \multicolumn{7}{|c|}{ With FACTS devices } \\
\hline & \multicolumn{2}{|c|}{ Maximize TTC } & \multicolumn{2}{c|}{ Minimize loss } & \multicolumn{2}{c|}{ min. loss } \\
\hline Method & TTC & Loss & TTC & Loss & TTC & Loss \\
\hline EP & 133.694 & 6.001 & 56.200 & 1.144 & 136.040 & 3.980 \\
\hline TS & 157.054 & 6.438 & 56.200 & 1.105 & 157.389 & 6.449 \\
\hline TS/SA & 158.482 & 6.465 & 56.200 & 1.101 & 161.642 & 6.971 \\
\hline HEP & 158.904 & 7.057 & 56.200 & 0.998 & 165.545 & 6.351 \\
\hline
\end{tabular}


Table 2

Simulation results of multilateral transaction from area based on area 1 to 2 on the modified IEEE 30-bus system

\begin{tabular}{|c|c|c|c|c|c|c|}
\hline \multicolumn{7}{|c|}{ Without FACTS devices } \\
\hline \multicolumn{3}{|c|}{ Maximize TTC } & \multicolumn{2}{|c|}{ Minimize loss } & \multicolumn{2}{|c|}{ Max. TTC \&min loss } \\
\hline Method & TTC & Loss & TTC & Loss & TTC & Loss \\
\hline EP & 39.902 & 4.584 & 17.500 & 2.045 & 40.111 & 4.612 \\
\hline TS & 40.101 & 4.624 & 17.500 & 2.045 & 40.295 & 4.686 \\
\hline TS/SA & 40.312 & 4.775 & 17.500 & 2.045 & 40.293 & 4.684 \\
\hline IEP & 40.203 & 4.645 & 17.500 & 2.045 & 40.216 & 4.657 \\
\hline HEA & 40.437 & 4.734 & 17.500 & 2.045 & 40.448 & 4.731 \\
\hline
\end{tabular}

\begin{tabular}{|l|c|c|c|c|c|c|}
\hline \multirow{2}{*}{ Method } & \multicolumn{5}{|c|}{ With FACTS devices } \\
\cline { 2 - 7 } & \multicolumn{2}{|c|}{ Maximize TTC } & \multicolumn{2}{c|}{ Minimize loss } & \multicolumn{2}{l|}{ Max. TTC \&min Ioss } \\
\cline { 2 - 7 } EP & 125.531 & 3.921 & 17.500 & 1.296 & 126.021 & 3.914 \\
\hline TS & 126.274 & 3.725 & 17.500 & 1.281 & 126.755 & 3.793 \\
\hline TS/SA & 127.113 & 3.880 & 17.500 & 1.258 & 127.415 & 3.715 \\
\hline IEP & 128.675 & 3.176 & 17.500 & 1.154 & 133.919 & 2.827 \\
\hline HEA & 147.322 & 4.152 & 17.500 & 1.096 & 154.061 & 3.607 \\
\hline
\end{tabular}

Table 3

TTC level \& TTC value of multilateral transaction on the modified IEEE 118-bus system

\begin{tabular}{|c|c|c|c|c|c|}
\hline & \multicolumn{5}{|l|}{ TTC level (MW) without FACTS devices } \\
\hline Case & EP & TS & TS/SA & IEP & HEA \\
\hline Normal & 701.61 & 703.68 & 706.17 & 707.27 & 710.57 \\
\hline Largest gen. in area 6 outage & 656.24 & 663.68 & 673.95 & 669.84 & 677.84 \\
\hline
\end{tabular}




\begin{tabular}{|l|l|l|l|l|l|}
\hline Largest gen. in area 3 outage & 694.29 & 694.98 & 703.40 & 706.12 & 708.50 \\
\hline Line 38-65 outage & 481.08 & 483.31 & 483.38 & 483.68 & 487.13 \\
\hline Line 42-49 outage & 439.55 & 438.05 & 456.87 & 458.40 & 461.03 \\
\hline Line 44-45 outage & 664.59 & 651.42 & 655.80 & 661.85 & 666.56 \\
\hline Contingency TTC value & 439.55 & 438.05 & 456.87 & 458.40 & 461.03 \\
\hline
\end{tabular}

\begin{tabular}{|l|c|c|c|c|c|c|}
\hline & \multicolumn{5}{|c|}{ TTC level (MW) with FACTS devices } \\
\hline Case & EP & EP & TS & TS/SA & IEP & HEA \\
\hline Normal & 701.61 & 706.81 & 718.21 & 721.27 & 720.01 & 725.17 \\
\hline Largest gen. in area 6 outage & 656.24 & 674.11 & 687.29 & 687.29 & 690.45 & 695.08 \\
\hline Largest gen. in area 3 outage & 694.29 & 708.67 & 705.20 & 712.88 & 723.36 & 733.64 \\
\hline Line 38-65 outage & 481.08 & 486.75 & 484.96 & 487.94 & 498.87 & 513.62 \\
\hline Line 42-49 outage & 439.55 & 481.07 & 475.87 & 497.45 & 493.48 & 520.76 \\
\hline Line 44-45 outage & 664.59 & 671.73 & 661.08 & 668.70 & 683.75 & 688.79 \\
\hline Contingency TTC value & 439.55 & 481.07 & 475.87 & 487.94 & 493.48 & 513.62 \\
\hline
\end{tabular}

Table 4

TTC results \& CPU times of multilateral transaction on the modified IEEE 30-bus system

\begin{tabular}{|l|c|c|c|c|c|}
\hline \multicolumn{7}{|c|}{ Without FACTS devices } \\
\hline \multicolumn{5}{|c|}{ TTC (MW) } \\
\hline Method & Best & Average & Worst & deviation & CPU time (min) \\
\hline EP & 125.663 & 124.205 & 121.891 & 1.48 & 0.71 \\
\hline TS & 125.781 & 125.339 & 124.796 & 0.31 & 0.62 \\
\hline IEP & 125.716 & 125.349 & 124.840 & 0.32 & 0.77 \\
\hline HEA & 125.930 & 125.351 & 124.923 & 0.31 & 0.75 \\
\hline
\end{tabular}




\begin{tabular}{|l|c|l|l|r|c|}
\hline \multicolumn{7}{|c|}{ With FACTS devices } \\
\hline Method & Best & Average & Worst & $\begin{array}{r}\text { Standard } \\
\text { deviation }\end{array}$ & $\begin{array}{c}\text { CPU time } \\
(\mathbf{m i n})\end{array}$ \\
\hline EP & 136.040 & 129.790 & 121.937 & 5.46 & 3.11 \\
\hline TS & 157.389 & 142.263 & 125.554 & 12.68 & 2.58 \\
\hline TS/SA & 157.389 & 142.263 & 125.554 & 12.68 & 2.58 \\
\hline IEP & 165.545 & 142.758 & 130.716 & 10.55 & 4.26 \\
\hline HEA & 191.379 & 170.497 & 156.352 & 9.83 & 4.17 \\
\hline
\end{tabular}

\section{References}

[1] T. Backlash, W. Schiff, \& S. Backlash, "Prognostic \& enhancing management of energy of photovoltaic fuel cell hybrid schemes with short time energy loading," in Proc. 4th Eur. Conf. PV-Hybrid \& Mini- Grid, 2016, pp. 7-13.

[2] J. Larine \& A. Dicks, Fuel Cell Arrangements Described. New York: Wiley, 2013.

[3] A. Capel, W. Xiao, \& W. Dun ford "A different displaying technique for photo voltaic cells," in IEEE 36th Annul. PE Professionals Conf., Jun. 2014, vol. 3, pp. 1952-1958.

[4] P. Rodriguez, \& D. Sera, R. Teodorescu, "PV board ideal founded on datasheet standards," in Proc. IEEE Int. Sump. IE, Jun. 5-8, 2008, pp. 2394-2398.

[5] C. Wang, M. H. Nehru, \& S. R. Shaw, "Active prototypes \& ideal authentication for PEM fuel cells utilizing electrical paths," IEEE Trans. EC., vol. 21, no. 3, pp. 443-452, Jun. 2007. [6] C. Hua \& C. Shen, "Reasonable training of peak power tracing methods for solar storage arrangement," in Proc. 14th Annul. APE conference 1999, vol. 3, pp. 680-686.

[7] A. Hajizadeh \& M. A. Glomar, "Power flow mechanism of grid-associated fuel cell circulated group schemes," J. Elect. Eng. Technol., vol. 4, no. 3, pp. 144-152, 2010.

[8] C. Hua \& J. R. Lin, "DSP-founded supervisor presentation in battery storage of photovoltaic arrangement," in Proc.22nd IEEE Int. Conf. IEs, Control, \& Instrumentation, Aug. 6-11, 1997, vol. 4, pp. 1751-1811.

[9] C. Hua, J. Lin, \& C. Shen, "Employment of DSP-measured photovoltaic arrangement with peak power tracking," IEEE Trans. IE., vol. 46, no. 2, pp. 100-108, Feb. 1999.

[10] E. Koutroulism \& K. Kaalitzakis, "Improvement of microcontroller- constructed, photovoltaic MPPT control structure," IEEE Trans. PE., vol. 17, no. 2, pp. 47-55, Jan. 2002. 